\title{
Graphoepitaxy of $\mathrm{CeO}_{2}$ on $\mathrm{MgO}$ and its application to the fabrication of $45^{\circ}$ grain boundary Josephson junctions of $\mathrm{YBa}_{2} \mathrm{Cu}_{3} \mathrm{O}_{7-x}$
}

\author{
C. A. Copetti, J. Schubert, A. M. Klushin, S. Bauer, W. Zander, and Ch. Buchal \\ Institut für Schicht- und Ionentechnik, Forschungszentrum Jülich (KFA), D-52428 Jülich, Germany \\ J. W. Seo \\ Institut für Festkörperforschung, Forschungszentrum Jülich (KFA), D-52428 Jülich, Germany \\ F. Sanchez \\ Departamento de Fisica Aplicada i Electronica, Universitat de Barcelona, Avda Diagonal 647, \\ Barcelona E-08028, Spain
}

M. Bauer

Physik Department E 10, TU München, James-Frank Strasse 1, 8046 Garching, Germany

(Received 12 December 1994; accepted for publication 26 June 1995)

\begin{abstract}
We communicate a detailed study of the epitaxial growth of $\mathrm{CeO}_{2}$ on $\mathrm{MgO}$. The key feature of the growth is the dependence of the in-plane orientation of the $\mathrm{CeO}_{2}$ epitaxial layer on the $\mathrm{MgO}$ surface morphology. Atomic force microscopic (AFM) measurements, $x$-ray analyses, as well as high-resolution transmission electron microscopy (HRTEM) investigations reveal that on rough substrates a cube-on-cube growth of $\mathrm{CeO}_{2}$ on $\mathrm{MgO}$ occurs while on smooth substrates the $\mathrm{CeO}_{2}$ unit cell is rotated around the surface normal by $45^{\circ}$ with respect to the $\mathrm{MgO}$ unit cell when the deposition rate is low $(\sim 0.3 \AA / \mathrm{s})$ during the first stages of growth. This growth mechanism can be used for a defined fabrication of $45^{\circ}$ grain boundaries in the $\mathrm{CeO}_{2}$ layer by controlling the surface roughness of the $\mathrm{MgO}$ substrate. This report demonstrates that these $45^{\circ}$ grain boundaries may be used to fabricate $\mathrm{YBa}_{2} \mathrm{Cu}_{3} \mathrm{O}_{7-x}$ Josephson junctions. (C) 1995 American Institute of Physics.
\end{abstract}

\section{INTRODUCTION}

Grain boundaries in crystalline high temperature superconductors (HTS) can act as Josephson junctions. Their application in electronic devices makes it necessary to produce grain boundaries in a controllable and reproducible way. This can be realized by the deposition of an $\mathrm{YBa}_{2} \mathrm{Cu}_{3} \mathrm{O}_{7-x}$ $(\mathrm{YBaCuO})$ thin film over a substrate step, ${ }^{1}$ by using bicrystals ${ }^{2}$ and by the biepitaxial method. ${ }^{3}$ In the biepitaxial method, a $45^{\circ}$ grain boundary is produced by the controlled change of the in-plane orientation of an epitaxial $\mathrm{YBaCuO}$ film.

Up to now, many different combinations of materials have been used in the biepitaxial process. The process includes the deposition of a seed layer, patlerning of the seed layer, and, subsequently, the deposition of a buffer layer and of the superconductor $\mathrm{YBaCuO}$. The $45^{\circ}$ grain boundary is formed at the boundary between the regions with and without seed layer.

This report communicates the dependence of the inplane orientation of $\mathrm{CeO}_{2}$ on $\mathrm{MgO}$ (100) with the morphology of substrate and the deposition rate. It can be used to produce well defined $45^{\circ}$ grain boundaries, without any additional seed layer.

The relationship of an epitaxial layer on a substrate is determined by the interfacial energy and the lattice mismatch, which leads to strain energy. The layer orients itself in such a way that the lattice mismatch is small and the interfacial energy is minimized. Depending on the nature of substrate and film one or more epitaxial relations can be possible. The cluster nucleation depends on the substrate temperature and deposition rate and consequently both parameters can determine the epitaxial relationship. Addition- ally, the substrate surface morphology can influence the inplane orientation of an epitaxial layer. Surface steps act as nucleation centers and can impose a certain in-plane orientation of the growing film. This mechanism is known as graphoepitaxy. Graphoepitaxially determined growth of oxides has been observed in the system $\mathrm{YBaCuO}$ on $\mathrm{MgO}$ and $\mathrm{YBaCuO}$ on yttria-stabilized zirconia (YSZ). Norton et al. and Pennycook et al. deduced from the fact that the interface between $\mathrm{YBaCuO}$ and $\mathrm{MgO}$ is incommensurable without localized strain fields that the epitaxial alignment of the $\mathrm{YBaCuO}$ grains is acquired at atomic steps. ${ }^{4}$ Recent results of one of the authors indicate on the other hand that this can also be explained by a rearrangement process during initial growth without any graphoepitaxial mechanism. ${ }^{5}$ Brorsson et al. showed that the in-plane alignment of $\mathrm{YBaCuO}$ on YSZ is sensitive to the surface morphology. Using YSZ substrates of different roughness they showed that the [110] $\mathrm{YBaCuO} / /[100] \mathrm{YSZ}$ orientation is promoted by a graphoepitaxial mechanism. ${ }^{6}$

$\mathrm{MgO}$ is a highly ionic insulating solid, crystallizing in the $\mathrm{NaCl}$ structure. It has low-energy, charge neutral (100) cleavage planes. The lattice constant of $\mathrm{MgO}$ is $4.2 \AA . \mathrm{CeO}_{2}$ has a cubic fluorite-type structure with a lattice constant of $5.4 \AA$. The smallest epitaxial misfit of $\sim 10 \%$ is achieved when the $\mathrm{CeO}_{2}$ unit cell is rotated around the surface normal by $45^{\circ}$ with respect to the $\mathrm{MgO}$ unit cell, i.e., with an inplane orientation: $\mathrm{CeO}_{2}[110] / \mathrm{MgO}[100]$.

In contrast, a nonrotated growth mode (cube-on-cube) with an in-plane orientation $\mathrm{CeO}_{2}[100] / \mathrm{MgO}[100]$ results in a misfit of about $22 \%$. However, a smaller misfit of $4 \%$ is calculated when the epitaxial growth is described in terms of domains, since three lattice parameters of $\mathrm{CeO}_{2}$ match with 

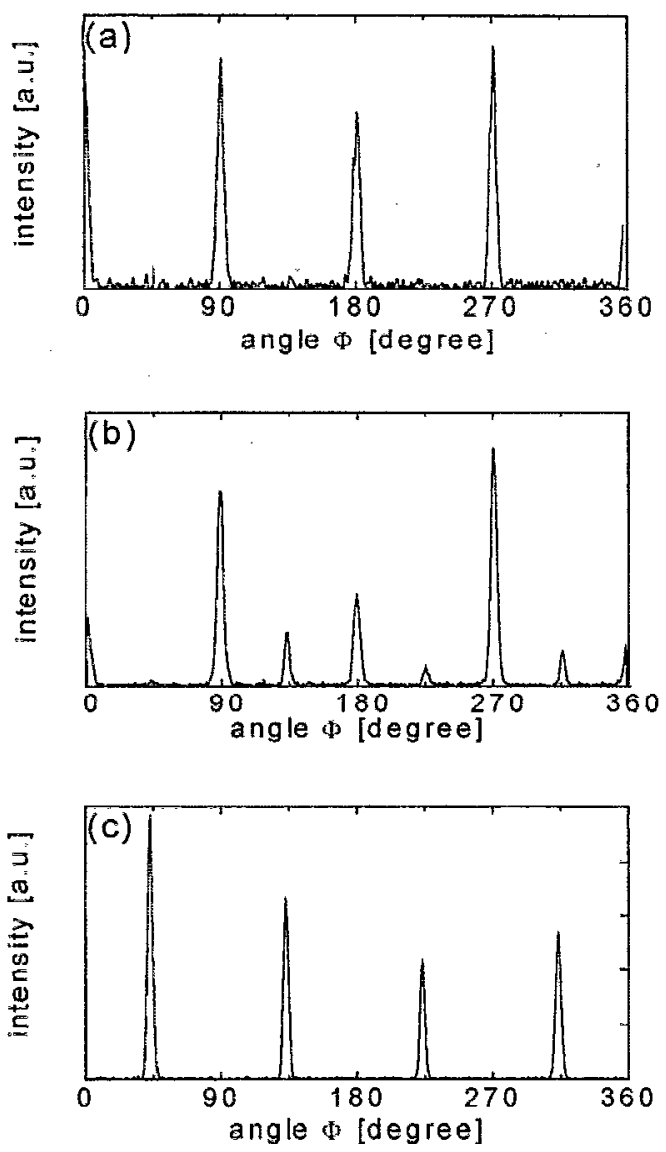

FIG. 1. $\mathrm{X}$-ray $\phi$ scans of $\mathrm{CeO}$, (420) on different (100) $\mathrm{MgO}$ substrates. (a) For a rough $\mathrm{MgO}$ substrate, (b) for a smooth substratc. $0^{\circ}$ corresponds to a [100] substrate direction being parallel to the diffracting plane.

four of the $\mathrm{MgO}$ substrates. Therefore, the lattice mismatch strain in this orientation is large but the one associated with the domains is comparatively small.

Cube-on-cube and a multi-in-plane orientation of $\mathrm{CeO}_{2}$ on $\mathrm{MgO}$ has been reported by many groups. ${ }^{3,7}$ To our knowledge there are no previous reports of $\mathrm{CeO}_{2}$ grown on $\mathrm{MgO}$ with a pure $45^{\circ}$ rotation. Here, we report on our ability to induce both orientations of the $\mathrm{CeO}_{2}$ on $\mathrm{MgO}$ in a controlled way. As a key factor, we regard the surface morphology of the substrate and the deposition rate during the first stages of growth.

\section{EXPERIMENT}

Thin films of $\mathrm{CeO}_{2}$ and $\mathrm{MgO}$ were deposited on $\mathrm{MgO}$ by electron beam evaporation from pellets of $\mathrm{CeO}_{2}$ or $\mathrm{MgO}$. The deposition was performed at an oxygen partial pressure of $1-2 \times 10^{-4}$ mbar. Optimum crystalline quality was achieved at a substrate heater temperature of $900^{\circ} \mathrm{C}$ for $\mathrm{CeO}_{2}$ and $525^{\circ} \mathrm{C}$ for $\mathrm{MgO}$.

We deposited $\mathrm{CeO}_{2}$ on three types of $\mathrm{MgO}$ substrates of different polishing, i.e., of different surface roughness.

The crystalline quality of the $\mathrm{CeO}_{2}$ films was studied by Rutherford backscattering spectrometry (RBS) and channeling, using $1.4 \mathrm{meV} \mathrm{He}^{+}$ions incident normal to the sample surface. The orientation was studjed by $x$-ray diffraction measurements. The surface quality was characterized by
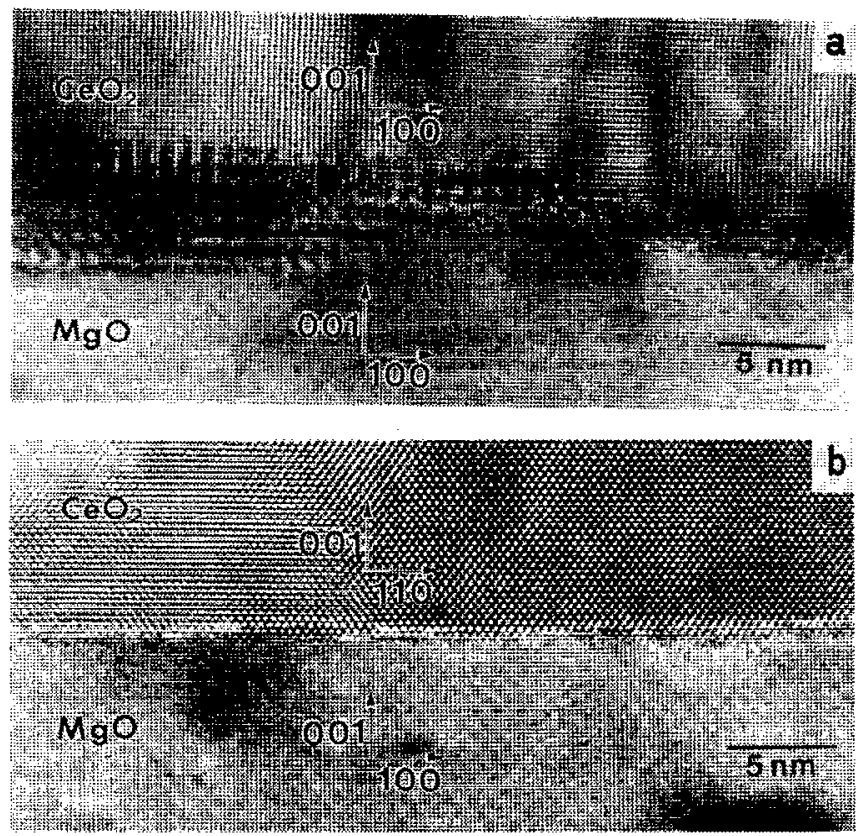

FIG. 2. Ctoss sectional lattice fringe images of two $\mathrm{CeO}_{2}$ films grown on a rough $\mathrm{MgO}$ substrate (a) and on a smooth substrate (b). The different orientations of the $\mathrm{CeO}_{3}$ with respect to the $\mathrm{MgO}$ are indicated by arrows.

cross sectional lattice fringe transmission electron microscopy (TEM) and noncontact atomic force microscopy. All $\mathrm{CeO}_{2}$ films were of high crystalline quality. A minimum yield in the channeling spectra in the [100] direction of less than $5 \%$ was measured. X-ray measurements of the $(004)$ $\mathrm{CeO}_{2}$ reflex showed a rocking curve width of $0.5^{\circ}$ to $1^{\circ}$, independent of the in-plane orientation of the film.

\section{RESULTS AND DISCUSSION}

The deposition of $\mathrm{CeO}_{2}$ on smooth $\mathrm{MgO}$ substrates without a controllable deposition rate does not ensure reproducibility. Cube-on-cube films are obtained when deposition rate in the first stage of deposition is high whereas the $45^{\circ}$ rotated growth occurs for low deposition rate. A shutter was placed to fix the deposition rate. A low deposition rate of about $0.3 \AA / s$ warrants $45^{\circ}$ rotated growth. A mixture of both orientations is observable when the deposition rate is higher. In the following we present investigations of $\mathrm{CeO}_{2}$ films grown with a low deposition rate of about $0.3 \ddot{A} / \mathrm{s}$.

Figure 1 shows x-ray diffraction $\phi$ scans of $\mathrm{CeO}_{2}(420)$ reflections of films deposited on the three different $\mathrm{MgO}$ substrates with different polishing. The spectra reveal different in-plane orientations of $\mathrm{CeO}_{2}$ on the different substrates: a pure cube-on-cube growth [Fig. 1(a)]; a mixture of a cubeon-cube and a $45^{\circ}$ rotated growth [Fig. 1(b)]; and a pure $45^{\circ}$ rotated growth occurs [Fig. 1(c)].

The cross sectional lattice fringe image of cube-on-cube oriented $\mathrm{CeO}_{2}$ on $\mathrm{MgO}$ [Fig. 2(a)] shows Moire fringes at the interface due to a superposition of $\mathrm{CeO}_{2}$ and $\mathrm{MgO}$ in the viewing direction. The spacing of these fringes is in accordance with the values calculated from the lattice parameters of $\mathrm{MgO}$ and $\mathrm{CeO}_{2}$. Therefore, it can be concluded that the interface of the cube-on-cube oriented $\mathrm{CeO}_{2}$ and $\mathrm{MgO}$ is 
wavy with a roughness up to $8 \mathrm{~nm}$. In contrast, the interface between the $45^{\circ}$ rotated $\mathrm{CeO}_{2}$ and the $\mathrm{MgO}$ substrate is very smooth [Fig. 2(a)]. No steps higher than one unit cell of MgO can be observed. This is confirmed by AFM measurements. Height variations of only 0.4 nm were measured. ${ }^{8}$

Substrates, where both $\mathrm{CeO}_{2}$ orientations were observed, have an intermediate roughness. We measured a typical height variation of $0.8 \mathrm{~nm}$ and higher by AFM.

Since all films were grown under the same conditions, we conclude that the orientation of the $\mathrm{CeO}_{2}$ films is strongly dependent on the surface morphology, too. Two competing in-plane orientations of $\mathrm{CeO}_{2}$ on [100] $\mathrm{MgO}$ are possible. The orientation $[110] \mathrm{CeO}_{2} / /[100] \mathrm{MgO}$ has a slightly lower interfacial energy than the $[100] \mathrm{CeO}_{2} / /[100] \mathrm{MgO}$ orientation. In the initial stage of the deposition of $\mathrm{CeO}_{2}$ on $\mathrm{MgO}$, nucleation can occur on terraces and at surface steps. If nucleation occurs on terraces, the growth of $[110] \mathrm{CeO}_{2}$ is favored due to the lower interfacial energy. In contrast, a nucleation at steps will result in a cube-on-cube orientation. Therefore the deposition rate, step height, and step density on the surface determine the in-plane orientation of $\mathrm{CeO}_{2}$ on $\mathrm{MgO}$ (100). Steps on $\mathrm{MgO}$ form an additional surface for the nuclei, which may change the interfacial energy in favor of the cube-on-cube growth. Therefore the step height and step density on the surface determine the in-plane orientation of $\mathrm{CeO}_{2}$ on $\mathrm{MgO}$.

Since the surface roughness has been identified as the decisive parameter for the in-plane alignment, we tried different surface treatments in order to control this orientation. A similar approach has been used by Chew et al. ${ }^{9}$ They changed the in-plane orientation of $c$-axis YBaCuO on (001) MgO substrates by a preceding low-temperature argon milling process. $\mathrm{YBaCuO}$ films grown on milled regions of the substrate showed a film axis rotated $45^{\circ}$ with respect to films grown on untreated regions. They speculated that the ion milling breaks the step structure of the surface. Therefore, the morphological driving force is lowered and the in-plane orientation of $\mathrm{YBaCuO}$ is switched from $\mathrm{YBaCuO}(100)$ to $\mathrm{YBaCuO}(110)$ parallel to (100) $\mathrm{MgO}$. Unfortunately, they could not reproduce their results. ${ }^{10}$

In contrast to Chew et al. we started with smooth substrates, and used several techniques to roughen the surface in order to change the orientation of $\mathrm{CeO}_{2}$. Both, Ar-ion milling $\left(500 \mathrm{~V}, 0.5 \mathrm{~mA} / \mathrm{cm}^{2}\right.$ for $\left.3 \mathrm{~min}\right)$ and Ce implantation $(50 \mathrm{keV}$ ions incident $50^{\circ}$ relative to the sample surface with doses ranging from $5 \times 10^{12}$ to $10^{15} / \mathrm{cm}^{2}$ ) resulted in a change in orientation of the $\mathrm{CeO}_{2}$ film. However, a residual amount of $5 \%$ to $10 \%$ of $45^{\circ}$ rotated grains in cube-on-cube aligned $\mathrm{CeO}_{2}$ was still observed. Therefore, both techniques need further improvements.

Another approach does not use any of the abovementioned surface treatments. Instead, the homoepitaxy of MgO on MgO substrates serves as a roughening step. The homoepitaxy of $\mathrm{MgO}$ proceeds via island growth. The high density of these islands reliably provides the necessary reference steps for the desired cube-on-cube growth. Figures $3(\mathrm{a})$ and 3 (b) show AFM measurements, which illustrate the change of the surface morphology of a smooth $\mathrm{MgO}$ surface due to the deposition of $40 \mathrm{~nm} \mathrm{MgO}$. The initially very
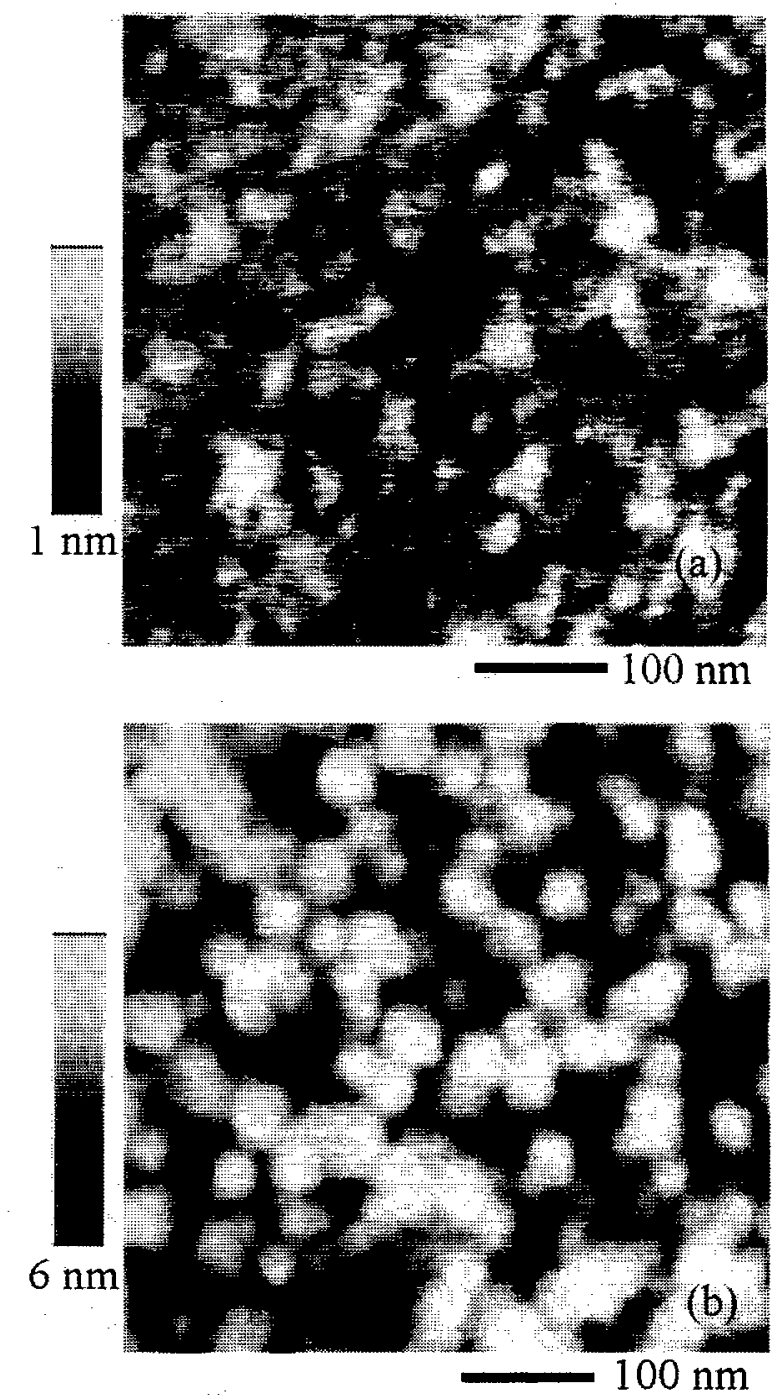

FIG. 3. AFM measurements of a smooth MgO substrate (a) and of a smooth substrate covered homoepitaxially with $40 \mathrm{~nm} \mathrm{MgO}$ (b). In (b) the edges of the picture are parallel to [100] directions of $\mathrm{MgO}$.

smooth substrate, with a height variation of about $0.4 \mathrm{~nm}$ [Fig. 3(a)] is changed into a rough surface, with a height variation of more than $3 \mathrm{~nm}$ [Fig. 3(b)]. The threedimensional growth of $\mathrm{MgO}$ on $\mathrm{MgO}$ leads to a very high density of rectangular islands, whose edges are oriented in the (100) direction. Therefore, graphoepitaxial nucleation of $\mathrm{CeO}_{2}$ at these steps determines the orientation of the $\mathrm{CeO}_{2}$

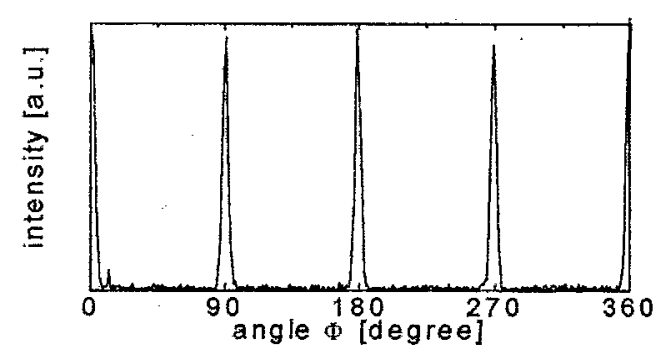

FIG. 4. X-ray $\phi$ scans of $\mathrm{CeO}_{2}$ (420) on a smooth (100) MgO substrate covered homoepitaxially with $40 \mathrm{~nm} \mathrm{MgO} .0^{\circ}$ corresponds to a [100] substrate direction being parallel to the diffracting plane. 


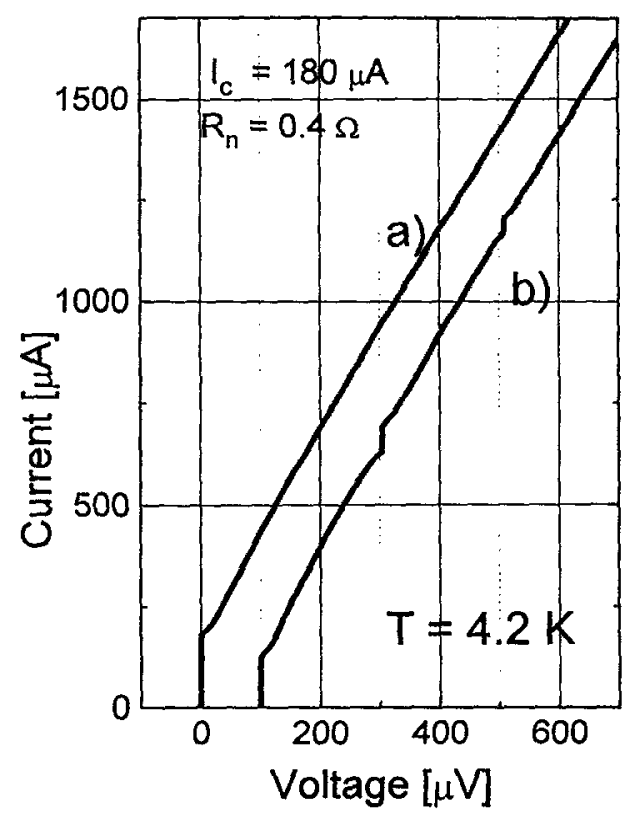

FIG. 5. IVC of a $10-\mu \mathrm{m}$-wide $45^{\circ}$ grain boundary junction at $4.2 \mathrm{~K}$ (a) without external if current and (b) with microwave irridation at $98.9 \mathrm{GHz}$. For clarity, curve (b) is displaced horizontally.

films. This is shown by the $\phi$ scan in Fig. 4. It corresponds to a $\mathrm{CeO}_{2}$ layer deposited on a smooth $\mathrm{MgO}$ substrate covered homoepitaxially with $40 \mathrm{~nm} \mathrm{MgO}$. Only reflexes belonging to a cube-on-cube orientation of $\mathrm{CeO}_{2}$ on $\mathrm{MgO}$ are observable.

For $45^{\circ}$ grain boundary Josephson junctions, both $\mathrm{CeO}_{2}$ orientations on one substrate are required. We achieved this goal by positioning a shadow mask over the substrate to cover half of it prior to the $\mathrm{MgO}$ deposition. The resulting $\mathrm{MgO}$ edge is smooth and flat. Thus, we expect that step edges do not form. After $\mathrm{MgO}$ deposition, the substrate is totally covered by $\mathrm{CeO}_{2}$. The $\mathrm{CeO}_{2}$ grows $45^{\circ}$ rotated on the smooth part of the substrate, while on the rough partcovered homoepitaxially with $\mathrm{MgO}$-it grows cube-on-cube. Therefore a $45^{\circ}$ grain boundary in $\mathrm{CeO}_{2}$ is formed at the boundary of both regions. This grain boundary is transferred into the $\mathrm{YBaCuO}$ film, grown on top of the $\mathrm{CeO}_{2}$ film.

Across these grain boundaries we patterned narrow bridges, using standard photolithography and ion milling.
Below $T_{c}$, these microbridges form Josephson junctions. A typical current voltage curve is depicted in Fig. 5. It shows a IV curve of a 10- $\mu$ m-wide and a 200 -nm-thick YBaCuO bridge containing the grain boundary at $4.2 \mathrm{~K}$ with (b) and without (a) microwave irridation $(f=100 \mathrm{GHz})$. At the expected voltage spacing of about $200 \mu \mathrm{V}$ well defined rfinduced steps are visible, proving the phase-locking of the Josephson oscillations with the external of radiation. The $I_{c} R_{n}$ products of the junctions are in the range of 30 to 90 $\mu \mathrm{V}$ at $4.2 \mathrm{~K}$, which is comparable with $I_{c} R_{n}$ products of $45^{\circ}$ grain boundary Josephson junctions on $\mathrm{MgO}$ substrates. ${ }^{3,9}$

\section{SUMMARY AND CONCLUSION}

In summary, we have shown that the in-plane orientation of $\mathrm{CeO}_{2}$ on $\mathrm{MgO}$ is determined by the deposition rate and the surface roughness of the $\mathrm{MgO}$ substrate. By depositing the film at low deposition rate during the first stages of growth and controlling the surface roughness it is possible to create $45^{\circ}$ grain boundaries in $\mathrm{CeO}_{2}$. These grain boundaries can be used to fabricate $\mathrm{YBaCuO}$ Josephson junctions.

\section{ACKNOWLEDGMENT}

One of the authors (F. S.) acknowledges the support of a grant from Direction General de Investigacion Cientifica y Tecnica.

${ }^{1}$ K. P. Daly, W. D. Dozier, J. F. Burch, S. B. Coons, R. Hu, C. E. Platt, and R. W. Simon, Appl. Phys. Lett. 58, 543 (1991).

${ }^{2}$ D. Dimos, P. Chaudari, J. Mannhart, and F. K. LeGoues, Phys. Rev. Lett. 61, 219 (1988).

${ }^{3}$ K. Char, M. S. Colclough, L. P. Lee, and G. Zaharchuk, Appl, Phys. Lett. 59, 2177 (1991).

${ }^{4}$ M. G. Norton and C. B. Carter, J. Cryst. Growth 110, 64 (1990); S. J. Pennycook, M. F. Chisholm, D. E. Jesson, R. Feenstra, S. Zhu, X. Y. Zheng, and D. J. Lowndes, Physica C 202, 1 (1992).

${ }^{5} \mathrm{M}$. Bauer, F. Baudenbacher, and $\mathrm{H}$. Kinder (unpublished).

${ }^{6}$ G. Brorsson, E. Olsson, Z. G. Ivanov, E. A. Stephantsov, J. A. Alarco, Yu.

Boikov, T. Claeson, P. Berastegui, V. Langer, and M. Löfgren, J. Appl. Phys. 75, 7958 (1994).

${ }^{7}$ X. D. Wu, L. Luo, R. E. Muenchausen, K. N. Springer, and S. Foltyn, Appl. Phys. Lett. 60, 1381 (1992).

${ }^{8}$ As height variation we define the range of heights in which $90 \%$ of the area in a $1 \mu \mathrm{m}$ times $1 \mu \mathrm{m}$ AFM image is found.

${ }^{9}$ N. G. Chew, S. W. Goodyear, R. G. Humphreys, J. S. Satchell, J. A. Edwards, and M. N. Keene, Appl. Phys. Lett. 60, 1516 (1992).

${ }^{10} \mathrm{~N}$. G. Chew (private communication). 